\title{
Escenarios territoriales para la gestión preventiva de la desertificación en la cuenca del Puangue, Chile
}

\author{
Ana Huaico Malhue ${ }^{1}$ ana.huaico@uabc.edu.mx, Claudia Leyva Aguilera ${ }^{1}$, \\ Nelly Calderón de la Barca ${ }^{1}$, Ileana Espejel ${ }^{1}$, Pilar Cereceda ${ }^{2}$
}

\begin{abstract}
RESUMEN
El presente trabajo propone diferentes escenarios territoriales para la toma de decisiones con el fin de prevenir los procesos de desertificación en la cuenca del Puangue en la Región Metropolitana de Santiago de Chile. Lo anterior se realiza a través de la aplicación de un sistema de indicadores de la peligrosidad ambiental a la desertificación a escala local. Como resultado se identifican en el territorio las áreas en peligro a desertificarse y se construye la proyección con cambio climático, concluyéndose en la necesidad de generar acciones para prevenir el avance de la desertificación. Este documento se convierte en una aproximación para el conocimiento integrado a escala local para la gestión preventiva de las zonas en peligro a desertificarse.
\end{abstract}

Palabras clave: desertificación, peligrosidad, escenarios, prevención, gestión.

\section{Spatial scenarios for the preventive management of desertification in the dasin Puangue, Chile}

\begin{abstract}
This paper proposes territorial scenarios for decision making in order to prevent desertification processes in the Puangue basin, in the Metropolitan Region of the Santiago, Chile. The above is done through the implementation of a system of indicators of environmental dangerousness for desertification in a local scale. As a result, the identified territory in areas at risk to desertification and the projection is constructed with climate change, concluding on the need to generate action to prevent further desertification. This document becomes an approach to integrated local knowledge for the preventive management of the areas at risk to desertification.
\end{abstract}

Key words: desertification, dangerous, stages, prevention and management.

Recibido el 24 de octubre de 2010, aceptado el 17 de mayo de 2011

\footnotetext{
1 Maestría en Ciencias en Manejo de Ecosistemas de Zonas Áridas, Facultad de Ciencias, Universidad Autónoma de Baja California, Carretera Tijuana-Ensenada km 106 apdo. postal 1880, Ensenada 22860 B.C. México.

2 Centro de Estudios del Desierto de Atacama, Pontificia Universidad Católica de Chile, Avenida Vicuña Mackenna 4860, Santiago, Chile.
} 


\section{INTRODUCCIÓN}

El presente trabajo muestra los posibles escenarios territoriales para la gestión preventiva de la desertificación en la cuenca del Puangue, una zona rural en la Región Metropolitana de Santiago de Chile; aunque la cuenca actualmente no se encuentra con áreas desertificadas, este trabajo demuestra que tiene altos índices de peligrosidad ambiental a la desertificación, por lo que se hace necesario llevar a cabo un plan de manejo preventivo. El diagnóstico se logra a partir de la confección de un índice de desertificación a escala local que cuenta con indicadores de sensibilidad ambiental y de presión, lo que es una herramienta útil para la toma de decisiones.

La desertificación se define como un proceso complejo que reduce la productividad y el valor de los recursos naturales en el contexto específico de condiciones climáticas áridas, semiáridas y subhúmedas secas, como resultado de las variaciones climáticas y a las acciones humanas adversas (UNCED, 1992). Según la Comisión Económica para América Latina y el Caribe (MORALES \& PARADA 2005), América Latina y el Caribe posee una superficie de 20.18 millones de $\mathrm{km} 2$, de los cuales un $25 \%$ corresponde a tierras áridas, semiáridas y subhúmedas secas. De este total, a su vez, el 75\%, es decir, alrededor de 378 millones de hectáreas, presenta serios problemas de degradación. A estos se suman otros ecosistemas afectados por el mismo tipo de problemas, con distinto grado de intensidad.

De acuerdo con MORALES \& PARADA (2005), en Chile al año 2000 la desertificación afectaba a 19 mil 286 unidades productivas correspondientes a 48.3 millones de hectáreas, impactando a 1.3 millones de personas, que equivalen al $22.1 \%$ del total de la población del país. De acuerdo con la Corporación Nacional Forestal (CONAF 2007), las causas profundas del fenómeno de la desertificación en Chile son el resultado en parte de actividades arraigadas culturalmente en la zona rural chilena como la deforestación de los terrenos forestales para el uso agropecuario, los incendios forestales, el sobre-pastoreo, el uso agropecuario de terrenos forestales, el uso inapropiado del riego, entre otras, siendo la deforestación la mayor causa de desertificación.

De acuerdo con DE PEDRAZA (2006), la persistencia en el tiempo de la mala gestión ha generado unos procesos de efectos acumulados altamente degradantes en los sistemas terrestres; el crecimiento exponencial de la población y la consecuente extensión de su actividad sobre el medio natural, prácticamente han universalizado los problemas. Finalmente, el desarrollo tecnológico ha implicado la intensificación del proceso anterior y la aparición de nuevas actividades que sí son intrínsecamente degradantes y como ocurre con la mayoría de los problemas ambientales, en el origen de la desertificación hay factores directamente relacionados con las actividades productivas.

En Chile, como en el resto de América Latina los estudios y las medidas de gestión de los procesos de desertificación se han centrado en medidas más bien correctivas que preventivas. Es por lo anterior que se propone una metodología de trabajo que va orientada a la gestión integrada para la prevención de los procesos de desertificación; con base en un escenario actual se generan escenarios tendenciales y estratégicos para la toma de decisiones a escala municipal en la cuenca del Puangue en la región mediterránea de Chile central.

Estudios e informes acerca de la desertificación, sus indicadores, su metodología y dimensiones que sirven de base para este trabajo son los que se encuentran en los trabajos de KOSMAS et al. (1999), MILLÁN et al. (2002), ABRAHAM (2003), MORALES \& PARADA (2005), REYNOLDS et al. (2005, 2007), MAESTRE et al. (2006) y FARAJZADEH \& EGBAL (2007). 
Con respecto a la construcción de escenarios, numerosas propuestas metodológicas la muestran como una herramienta válida para la toma de decisiones. En temas de desarrollo y medio ambiente los planteamientos de GÓMEZ OREA (2001), la EUROPEAN ENVIRONMENT AGENCY (EEA 2001) y el MILLENNIUM ECOSYSTEM ASSESSMENT (2005), sirven de referencia para la construcción de los escenarios para la gestión preventiva de los procesos de desertificación en la cuenca del Puangue. Por otro lado la Ley 19.300 de Bases Generales del Medio Ambiente, promulgada en 1994 por el Congreso Nacional de Chile, menciona que la prevención se encuentra dentro del
Art. 2, que define a la Protección del Medio Ambiente como el conjunto de políticas, planes, programas, normas y acciones destinados a mejorar el medio ambiente y a prevenir y controlar su deterioro.

\section{Área de Estudio}

La cuenca del Valle del Puangue se localiza en las estribaciones de la cordillera de la costa de Chile central, en la Región Metropolitana, con una superficie aproximada a los 1.841 kilómetros cuadrados, teniendo como drenaje principal el Estero Puangue (Fig.1), siendo los municipios en estudio, Curacaví, María Pinto y el sector de Mallarauco, en la comuna de Melipilla.

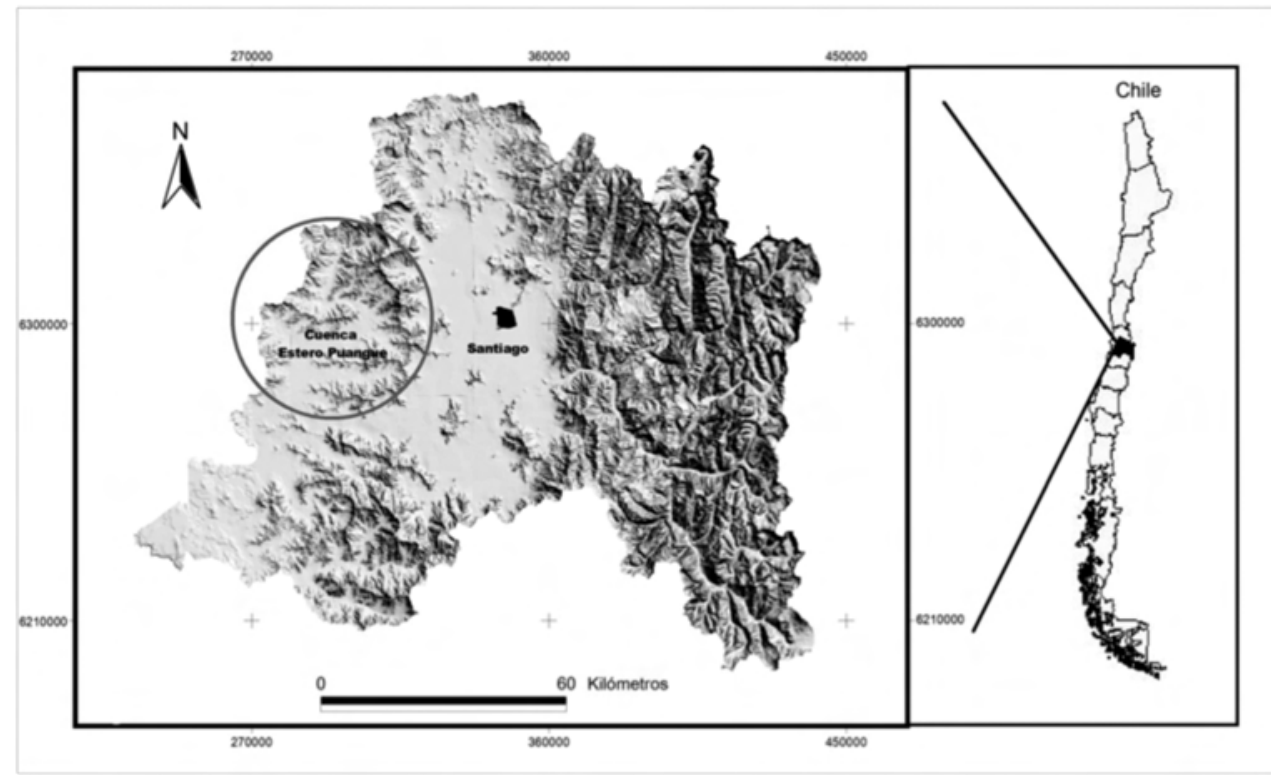

Fig. 1: Ubicación del área en estudio en la Región Metropolitana de Santiago.

El área de estudio se ubica dentro de los tres subsectores climáticos que separan la vertiente oriental de la Cordillera de la Costa (CIREN-CORFO 1990), más seca y calurosa que la occidental presente en la Región de Valparaíso, debido a su propia sombra pluviométrica y al abrigo de la moderación térmica costera. Este subsector recibe la influencia costera, pero fundamentalmente desarrolla condiciones climáticas semiáridas reforzadas por la cordillera de la costa.

En el área, el promedio de precipitaciones en la cuenca media (María Pinto) no supera los $450 \mathrm{~mm}$, lo que hace posible un paisaje semiárido en donde predomina principalmente la vegetación de matorral 
y matorral arborescente. En los últimos años la zona ha desarrollado un gran despliegue de actividades agrícolas, sobre todo en cultivos industriales (25.82\%, Censo Agropecuario, 2007), lo que ha demandado mayor cantidad de recursos naturales (suelo y agua especialmente).
Existe una creciente preocupación pública y privada por determinar qué tanto esto ha repercutido o puede repercutir, en la disponibilidad futura de estos recursos y que tan cerca se está de un nivel de degradación/desertificación de los suelos en la zona (Fig. 2).

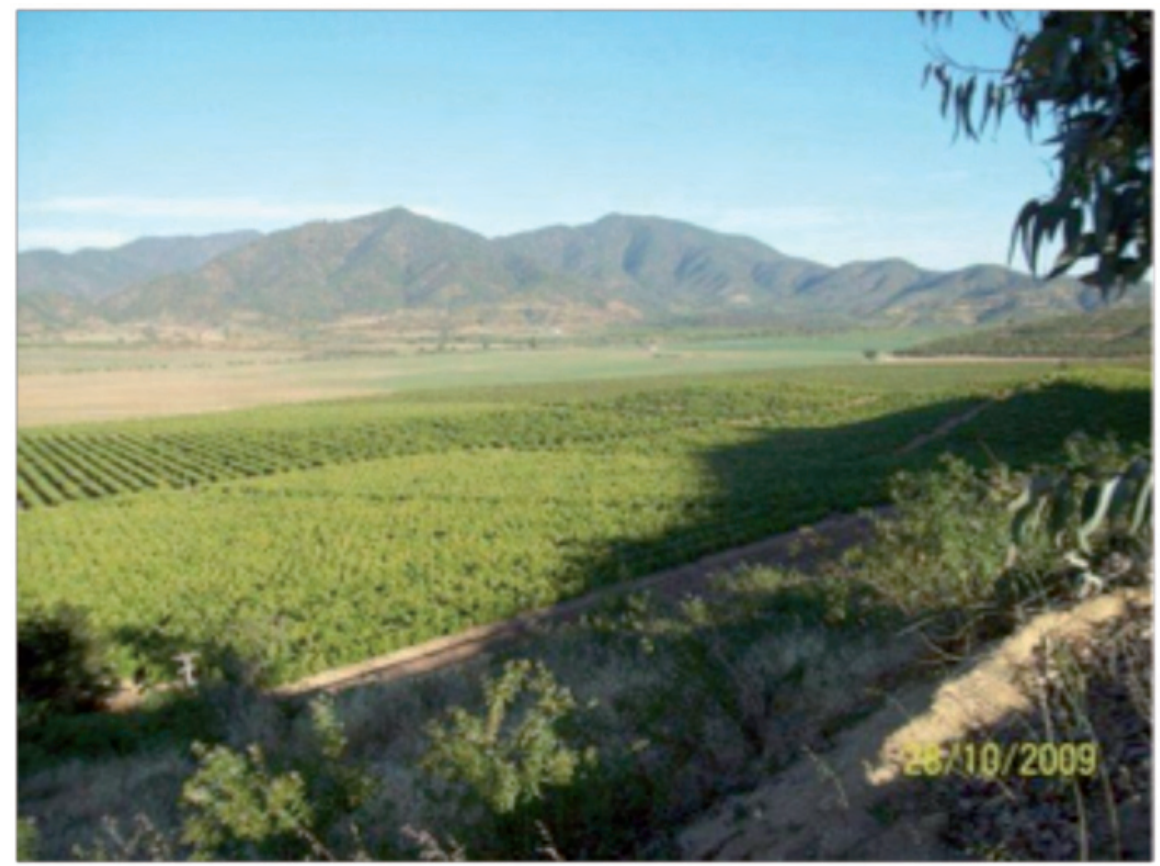

Fig. 2: Plantaciones industriales en la cuenca (Fuente: C. Huaico, octubre de 2009).

\section{MATERIALES Y MÉTODOS}

Los indicadores fueron escogidos tras la revisión bibliográfica y con base en la observación de campo de los procesos de desertificación en la cuenca del Puangue. La escala de trabajo, la disponibilidad de información y las características locales del área no permitieron la aplicación directa de alguno de los métodos que han sido aplicados en otros lugares en el mundo. En este contexto y con base en el modelo conceptual Presión, Estado, Respuesta (PER, OECD, 2003), se evaluó el estado de la cuenca a través de la aplicación de la metodología para la evaluación de áreas sensibles a la desertificación en zonas mediterráneas planteada por KOSMAS et al. (1999), estimándose la calidad de: suelo, clima, vegetación, uso del suelo y gestión, así como también, se identificaron los indicadores de presión (2007), por lo que se unen ambas metodologías para obtener el índice de peligrosidad ambiental a la desertificación.

\section{Sensibilidad ambiental a la desertificación}

La sensibilidad ambiental es definida como el resultado de las interacciones entre los factores elementales (capas de información) vinculados directa o indirectamente al fenómeno de degradación o desertificación, 
por lo que las áreas sensibles a desertificarse son pequeñas áreas que proveen de funciones ambientales críticas dentro del paisaje general, por lo que proteger estas funciones ecológicas vitales es considerado esencial para mantener la integridad y resiliencia del ecosistema (GEESON et al. 2002). El índice utilizado en este trabajo tiene como base la metodología planteada en el proyecto MEDALUS, que es un índice combinado con indicadores biofísicos y de gestión en los que algunos fueron adaptados a las condiciones del área de estudio, que incluye los indicadores presentados en la Tabla 1.

\section{Indicadores de presión}

Según el Instituto Nacional de Geografía y Estadística (INEGI 2006), estos indicadores describen la presión ejercida por las actividades humanas sobre el medio ambiente y los recursos naturales, reflejando intensidades de uso de recursos naturales y emisiones al ambiente, por lo que con base en los diversos trabajos en la materia se elabora un sistema de indicadores de presión.

En este contexto se consideró la información más reciente entre los años 2000-2010, contrastable en su caso con datos censales de los años 1960 e información cartográfica del año 1955 para el cálculo de las tasas de deforestación, y que contiene los siguientes elementos:
1) Déficit de distribución de agua aceptable (EscalaMunicipal,obtenida de la encuesta de Caracterización Socioeconómica del Gobierno de Chile, CASEN 2006)

2) Porcentaje de pobreza (Escala Municipal, obtenida de la encuesta CASEN 2006)

3) Tasa de crecimiento intercensal de población total 1960-2002

4) Tasa de crecimiento intercensal de población urbana (INSTITUTO NACIONAL DE ESTADÍSTICAS, Censos 1960 y 2002).

Cabe señalar que hubo una modificación administrativa entre los años 1960-2002, por lo que se redistribuyeron las localidades, ajustándolas a la actual distribución administrativa del área en estudio. La tasa de crecimiento intercensal de población se obtiene al relacionar una población en dos momentos, asumiendo un modelo de comportamiento, en este caso geométrico, de la forma:

$$
\mathrm{r}=[(\mathrm{Nx} / \mathrm{N} 0) 1 / \mathrm{t}-1]
$$

Donde:

$\mathrm{r}=$ Tasa de crecimiento intercensal

No $=$ Población en el año 0

$\mathrm{Nx}=$ Población en el año $\mathrm{X}$

$\mathrm{t}=$ Tiempo transcurrido en el periodo $(0-\mathrm{X})$

TABla i. Indicadores e Índices de CALIDAd (AdAPTAdo de KOSMAS et al. I999)

\begin{tabular}{|c|c|c|c|c|}
\hline Variable & Elemento & Indicador & & Índice de Calidad \\
\hline Suelo & Textura & Descripción & & \\
\hline & & (Arena, limo, arcilla) & Indicador & \\
\hline & & Bueno & 1 & \\
\hline & & Moderado & 1.2 & \\
\hline & & Poroso & 1.6 & \\
\hline & & Muy poroso & 2 & \\
\hline & Pendientes & & & \\
\hline & & $(\%)$ & Indicador & \\
\hline & & Muy suave a plano $<6$ & 1 & \\
\hline & & Suave 6 a 18 & 1.2 & \\
\hline
\end{tabular}




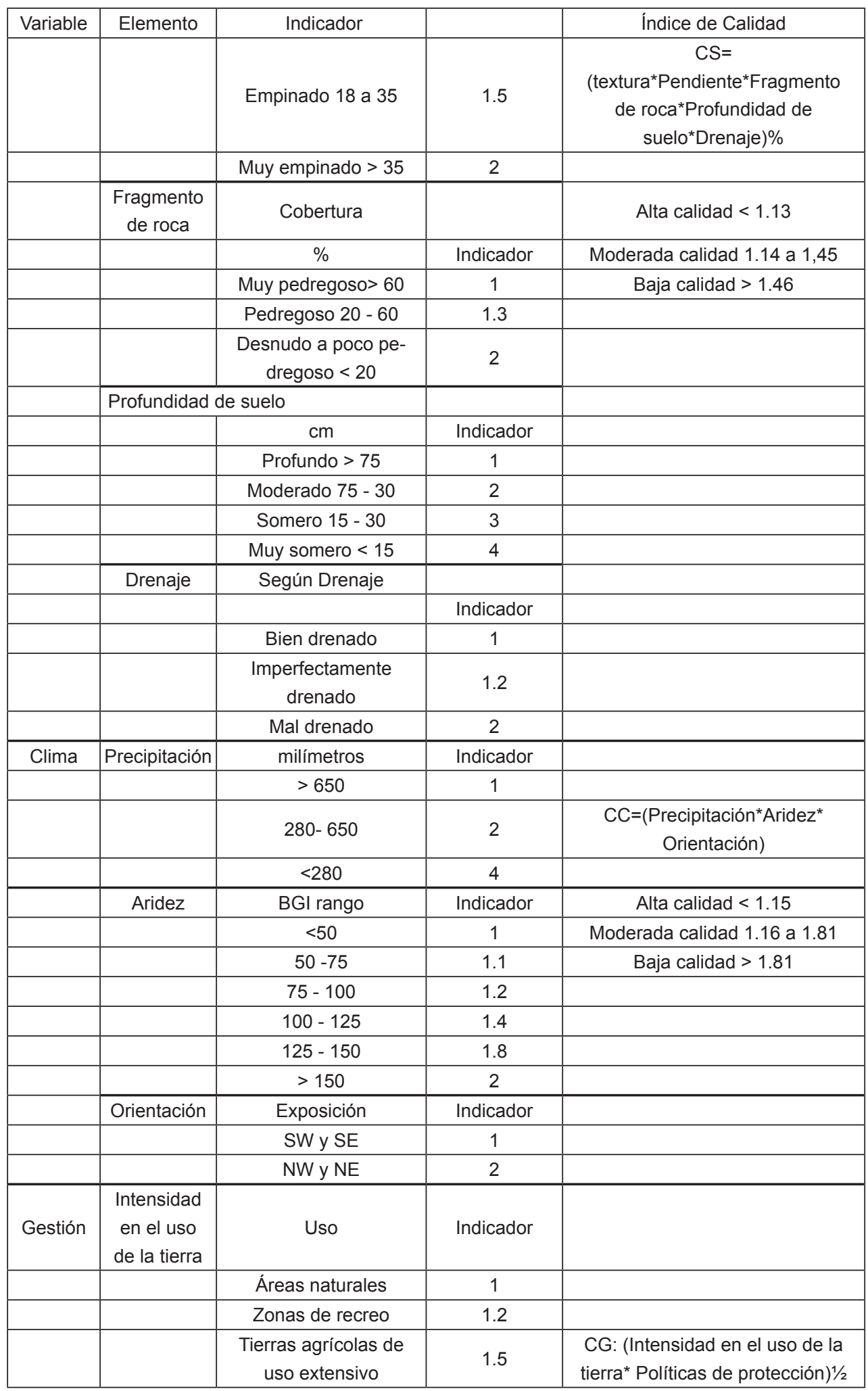




\begin{tabular}{|c|c|c|c|c|}
\hline Variable & Elemento & Indicador & & Índice de Calidad \\
\hline & & $\begin{array}{c}\text { Tierras agrícolas de } \\
\text { uso intensivo }\end{array}$ & 1.8 & \\
\hline & & Minería & 2 & Alto: 1 a 1.25 \\
\hline & & Zonas Urbanas & 2 & Moderado: 1.26 a 1.15 \\
\hline & Políticas & Grado de regulación & Indicador & Bajo: > 1.51 \\
\hline & & $\begin{array}{l}\text { Completo: }>75 \% \text { del } \\
\text { área bajo protección }\end{array}$ & 1 & \\
\hline & & $\begin{array}{l}\text { Parcial: } 25 \text { al } 75 \% \text { del } \\
\text { área bajo protección }\end{array}$ & 1.5 & \\
\hline & & $\begin{array}{c}\text { incompleto: }<\text { al } 25 \% \\
\text { del área bajo protec- } \\
\text { ción }\end{array}$ & 2 & \\
\hline & $\begin{array}{l}\text { Riesgo de } \\
\text { Incendio }\end{array}$ & Tipo de vegetación & Indicador & \\
\hline & & Tierra desnuda & 1 & \\
\hline & & $\begin{array}{c}\text { Cultivos agrícolas } \\
\text { anuales }\end{array}$ & 1.3 & \\
\hline & & Matorral y praderas & 1.6 & $\begin{array}{l}\text { CV: (Riesgo incendio* Protección } \\
\text { a la erosión* resistencia a la } \\
\text { sequía*cobertura) } 1 / 4\end{array}$ \\
\hline & & $\begin{array}{c}\text { Matorral arborescente, } \\
\text { matorral de suculen- } \\
\text { tas, bosque nativo }\end{array}$ & 2 & \\
\hline & $\begin{array}{l}\text { Protección } \\
\text { a la erosión }\end{array}$ & Tipo de vegetación & Indicador & Alto: 1 a 1.6 \\
\hline & & $\begin{array}{c}\text { Bosque nativo, ma- } \\
\text { torral arborescente, } \\
\text { matorral de suculentas }\end{array}$ & 1 & Moderado: 1.7 a 3.7 \\
\hline & & Matorral y Praderas & 1.3 & Bajo: > 3.8 a 16 \\
\hline & & Cultivos agrícolas & 1.6 & \\
\hline & & Tierra desnuda & 2 & \\
\hline & $\begin{array}{l}\text { Resistencia } \\
\text { a la sequía }\end{array}$ & Tipo de vegetación & & \\
\hline & & $\begin{array}{l}\text { Matorral, matorral de } \\
\text { suculentas, Matorral } \\
\text { arborescente, bosque } \\
\text { nativo }\end{array}$ & 1 & \\
\hline & & Terrenos agrícolas & 1.6 & \\
\hline & & Praderas & 1.8 & \\
\hline & Cobertura & Cobertura vegetal & & \\
\hline & & $>40$ & 1 & \\
\hline & & 10 a 40 & 1.8 & \\
\hline & & $<10$ & 2 & \\
\hline
\end{tabular}


La tasa de deforestación fue calculada a escala local, con base en el proyecto del Gobierno Regional, ORDENAMIENTO TERRITORIAL AMBIENTALMENTE SUSTENTABLE (1999) y con las ortofotos rectificadas del año 1955 (SAF 1956) a través de la siguiente fórmula (GUERRA \& OCHOA 2006).

r: $1-[1-(\mathrm{A} 1-\mathrm{A} 2 / \mathrm{A} 1] 1 / \mathrm{t}$

A1: Superficie forestal en el año 1
A2: Superficie forestal en el año 2

t: número de años entre las dos fechas

Ante la falta de información acerca del tema, los indicadores locales fueron contrastados con la información nacional, asignándole diferentes valores si estaban sobre o bajo los 0.5 grados porcentuales de los promedios nacionales, como lo muestra la Tabla 2 .

\section{TABLA 2. INDICADORES DE PRESIÓN}

\begin{tabular}{|c|c|}
\hline \multicolumn{2}{|l|}{ Distribución de Agua Aceptable (DDA) } \\
\hline Mayor o igual a 0.5 grados porcentuales al promedio nacional & 1 \\
\hline 0.49 grados porcentuales superior o inferior al promedio nacional & 2 \\
\hline Bajo o igual a 0.5 grados porcentuales al promedio nacional & 3 \\
\hline \multicolumn{2}{|l|}{ Porcentaje de pobreza $(P)$} \\
\hline Mayor o igual a 0.5 grados porcentuales al promedio nacional & 1 \\
\hline 0.49 grados porcentuales superior o inferior al promedio nacional & 2 \\
\hline Bajo o igual a 0.5 grados porcentuales al promedio nacional & 3 \\
\hline \multicolumn{2}{|l|}{ Tasa de Crecimiento de Población Total (TCPT) } \\
\hline Mayor o igual a 0.5 grados porcentuales a la tasa nacional & 3 \\
\hline 0.49 grados porcentuales superior o inferior a la tasa nacional & 2 \\
\hline Bajo o igual a 0.5 grados porcentuales a la tasa nacional & 1 \\
\hline \multicolumn{2}{|l|}{ Tasa de Crecimiento de Población Urbana (TCPU) } \\
\hline Mayor o igual a 0.5 grados porcentuales a la tasa nacional & 3 \\
\hline 0.49 grados porcentuales superior o inferior a la tasa nacional & 2 \\
\hline Bajo o igual a 0.5 grados porcentuales a la tasa nacional & 1 \\
\hline \multicolumn{2}{|l|}{ Tasa de Deforestación (TD) } \\
\hline Mayor o igual a 0.5 grados porcentuales a la tasa nacional & 3 \\
\hline 0.49 grados porcentuales superior o inferior a la tasa nacional & 2 \\
\hline Bajo o igual a 0.5 grados porcentuales a la tasa nacional & 1 \\
\hline
\end{tabular}

Es así como se obtiene el índice de presión a través de la siguiente fórmula: $\mathrm{IP}=(\mathrm{DAA}+\mathrm{P}+\mathrm{TCPT}+\mathrm{TCPU}+\mathrm{TD})^{1 / 5}$
Con lo que a nivel local es posible obtener municipios con diferentes tipos de presión (Tabla 3): 
TABLA 3. Tipos de PRESIón E Índice.

\begin{tabular}{|c|c|}
\hline Tipos de presión & Índice \\
\hline Presión alta & $\geq 1.67$ \\
\hline Presión Media & $\geq 1.48-\leq 1.66$ \\
\hline Presión Baja & $\leq 1.47$ \\
\hline
\end{tabular}

\section{Índice de peligrosidad ambiental}

Peligrosidad ambiental se refiere a todos los posibles riesgos que enfrenta la sociedad humana por los acontecimientos que se originan y transmiten a través del medio ambiente (SMITH et al. 2008). Este índice fue obtenido a partir de la suma del peso de la sensibilidad ambiental de la zona (AMS), y el peso otorgado a la unidad territorial según el índice de presión (IP) presentado por el municipio al cual pertenece la unidad AMS; luego el resultado de la suma de los dos primeros (AMS y IP), se multiplica por un valor de 3 si es de tipo crítico, 2 si es frágil y 1 si es de tipo potencial, dándole una mayor ponderación al tipo de sensibilidad ambiental que presenta (Fig. 3).

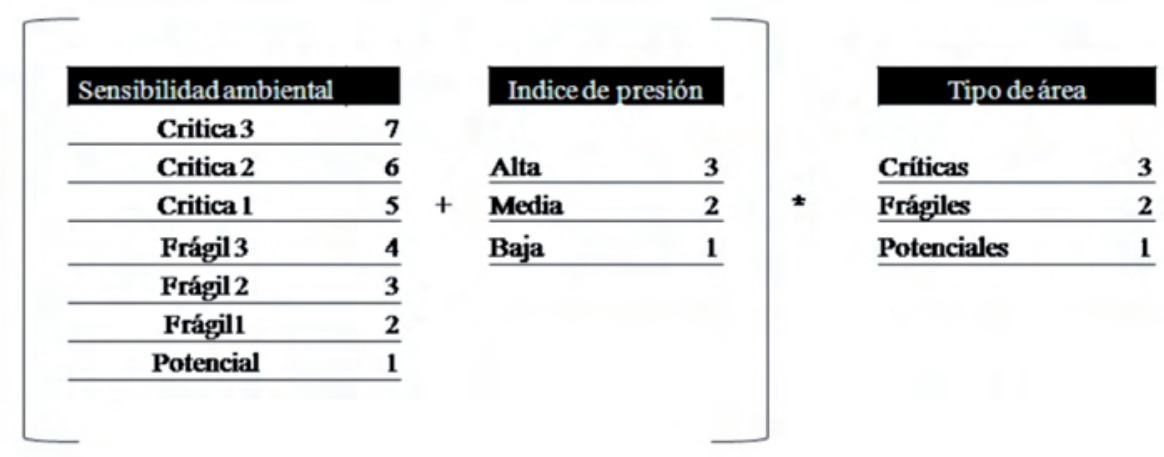

Fig. 3: Construcción del índice de peligrosidad ambiental.

Finalmente de todas las posibles el número de categorías de peligrosidad combinaciones de resultados se toma el que se quiere obtener, que en este caso son valor más alto y el más bajo, y se divide por $\quad$ cinco (tabla 4):

Tabla 4: Índice de Peligrosidad ambiental

\begin{tabular}{|c|c|}
\hline Peligrosidad & Índice \\
\hline Muy alta & $>25-\leq 30$ \\
\hline Alta & $>19-\leq 24$ \\
\hline Media & $>13-\leq 18$ \\
\hline Baja & $>6-\leq 12$ \\
\hline Muy baja & $\leq 6$ \\
\hline
\end{tabular}


A fin de sistematizar la información, se crea una tabla dinámica en una planilla de cálculo que proporciona automáticamente, dependiendo del tipo de sensibilidad ambiental de la unidad territorial y la presión, el tipo de peligrosidad a la desertificación que presenta el municipio.

\section{Definición de los escenarios}

De acuerdo con GÓMEZ OREA (2001), se denomina escenario a la descripción de una situación y el encadenamiento coherente de sucesos que partiendo de la situación actual llega a la futura. En este estudio se trabaja con base en tres escenarios: el actual que es la situación existente en el área de estudio, el tendencial que describe el escenario probable si no se efectúa ninguna intervención, en el que se fía el futuro al libre juego de la dinámica actual y el estratégico o de concertación, que es la situación más viable considerando las circunstancias que concurren en el sistema (GÓMEZ OREA 2001).

La situación tendencial contempla el futuro sin medidas de manejo, bajo la hipótesis de que continuarán las actuales tendencias de los indicadores de la zona y que la temperatura aumentará $0,2{ }^{\circ} \mathrm{C}$ por decenio (INTERGOVERNMENTAL PANEL ON CLIMATE CHANGE 2007). De tal forma que utilizando la metodología anterior (Indicadores AMS eIP) se estimó la situación futura y se obtuvo el escenario tendencial según el modelo antes descrito para 50 años, tal como lo plantea la EUROPEAN ENVIRONMENT AGENCY (2001), que dice que los escenarios científicos examinan el posible comportamiento de largo alcance de los sistemas biofísicos perturbados por la influencia humana, conociendo los aspectos actuales y del pasado.

El escenario estratégico o de concertación, se elabora bajo el supuesto que con un manejo adecuado se atenúa en un grado el peligro, disminuyendo así la tendencia a desertificarse. Sin embargo, cabe señalar que en este escenario se prevé que las zonas bajas y muy bajas se mantengan bajo el escenario actual.

\section{RESULTADOS}

\section{Los escenarios para la toma de decisiones}

El escenario actual es el reflejo de la historia de ocupación del valle, que en los últimos años ha sido altamente intervenido producto de las actividades agrícolas, por lo que la tasa de deforestación es alta en la cuenca en comparación a las tasas nacionales. Es así como en el año 1955 la zona poseía solamente una superficie de 24.027 hectáreas de suelo con uso agrícola, y al año 2002 esta cifra se duplicó a 51.907 hectáreas aproximadamente.

Los datos de crecimiento poblacional también son un reflejo de los procesos migratorios desde las áreas rurales, por lo que la población se ha concentrado en las zonas urbanas; lo anterior también es un reflejo de la aplicación de los instrumentos de planificación territorial como lo son los Planes Reguladores (DDU 227, respecto de la formulación y ámbito de acción de planes reguladores comunales), que han tendido a concentrar las viviendas sociales en las zonas urbanas principalmente por la factibilidad de dotar de servicios básicos, por lo que no es extraño que la zona posea altos índices de población con distribución de agua aceptable y, pese a que la tendencia es a la concentración de la población, esto no necesariamente indica un cambio en el desarrollo de las actividades productivas de la zona hacia actividades terciarias, sino que sigue siendo la actividad primaria agrícola la mayor fuerza de trabajo (tabla 5).

La situación de pobreza en el área en dos de los municipios estudiados es menor que el promedio nacional, sin embargo el municipio de María Pinto es el que se presenta con población más pobre, lo que 
repercute en la forma de relacionarse con su medio, ya que este tipo de indicador se vincula con una alta demanda de recursos energéticos provenientes de la vegetación nativa, ya que esta última es utilizada como combustible.

\section{Tabla 5. Municipios e indicadores. Encuesta CASEN (2006), InSTITUTO NACIONAL de EsTadísticas.}

\begin{tabular}{|c|c|c|c|c|}
\hline Municipios & Curacaví & María Pinto & Melipilla & Región/país \\
\hline Indicadores & & & & \\
\hline Sin distribución del agua aceptable (\%) & 1.8 & 1.4 & 3.7 & 1.7 \\
\hline Porcentaje de población pobre (\%) & 8.6 & 12.7 & 9.8 & 10.6 \\
\hline Tasa de crecimiento de población & 2.93 & 1.5 & 2.8 & 2.3 \\
\hline Tasa de crecimiento de población urbana & 6.08 & 6.46 & 5.6 & 3.5 \\
\hline Deforestación & 1.5 & 1.48 & 1.47 & 0.4 \\
\hline
\end{tabular}

Por otro lado, la cuenca posee 33.975 hectáreas altamente sensibles a desertificación, sobre todo aquellas que se encuentran en las terrazas del Estero Puangue y que han sido ocupadas durante décadas para uso agrícola; a lo anterior se le une la alta deforestación de vegetación nativa de las laderas de los cerros que han sido plantadas con cultivos, especialmente cítricos, paltas y viñedos, y que muchas veces no cuentan con el manejo adecuado para evitar procesos erosivos, que son los que repercuten aguas abajo depositando sedimentos en los suelos de mejor calidad que se encuentran en las terrazas del Puangue (Fig. 4).

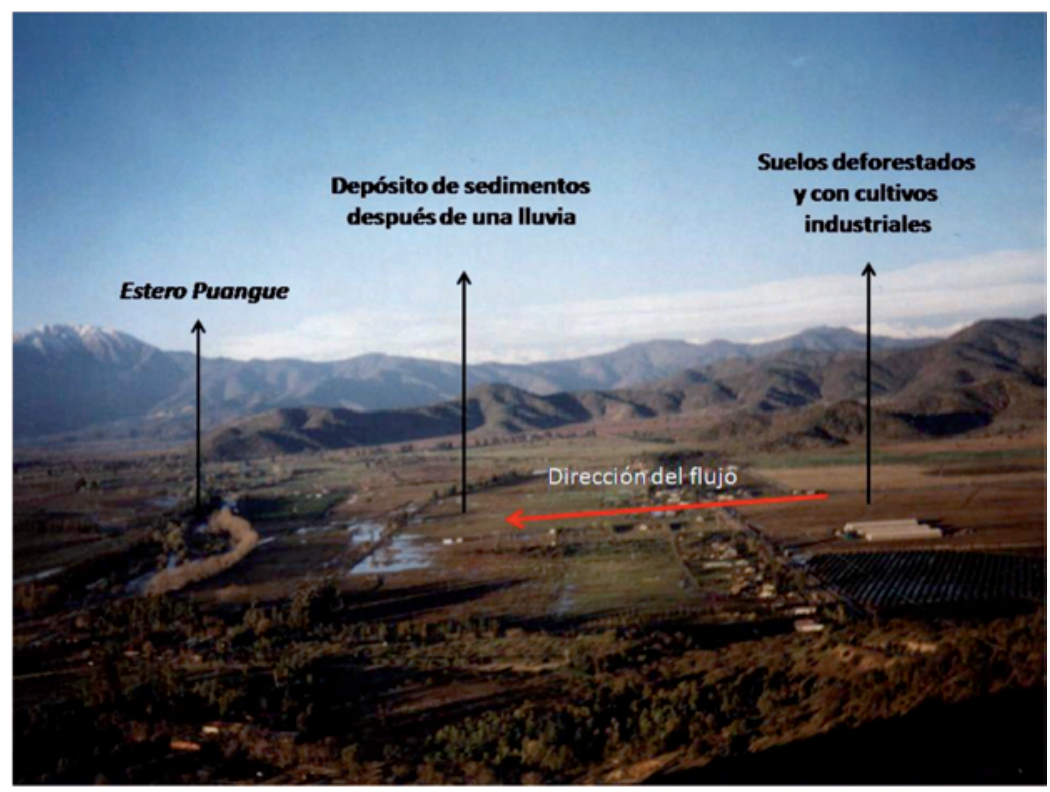

Fig. 4: Dinámica espacial ante un evento de lluvia en la localidad de Ranchillo, un sector del Municipio de María Pinto. Fotografía año 2002 de A. Huaico. 
Las zonas de peligro medio y alto son áreas de más reciente ocupación agrícola, donde juegan un papel fundamental factores como la calidad de suelo que indica suelos de buena calidad y profundidad, mientras que las zonas de peligrosidad bajas a muy bajas corresponden a suelos principalmente con vegetación de matorral, cuya mayor sensibilidad radica en el riesgo de que se produzcan incendios, y a las características climáticas de la cuenca que la hacen poseer un alto estrés hídrico. La figura 5 refleja cómo la cuenca aún no posee aparentemente áreas desertificadas, pero sí altamente sensibles, con zonas con un muy alto peligro a desertificarse.

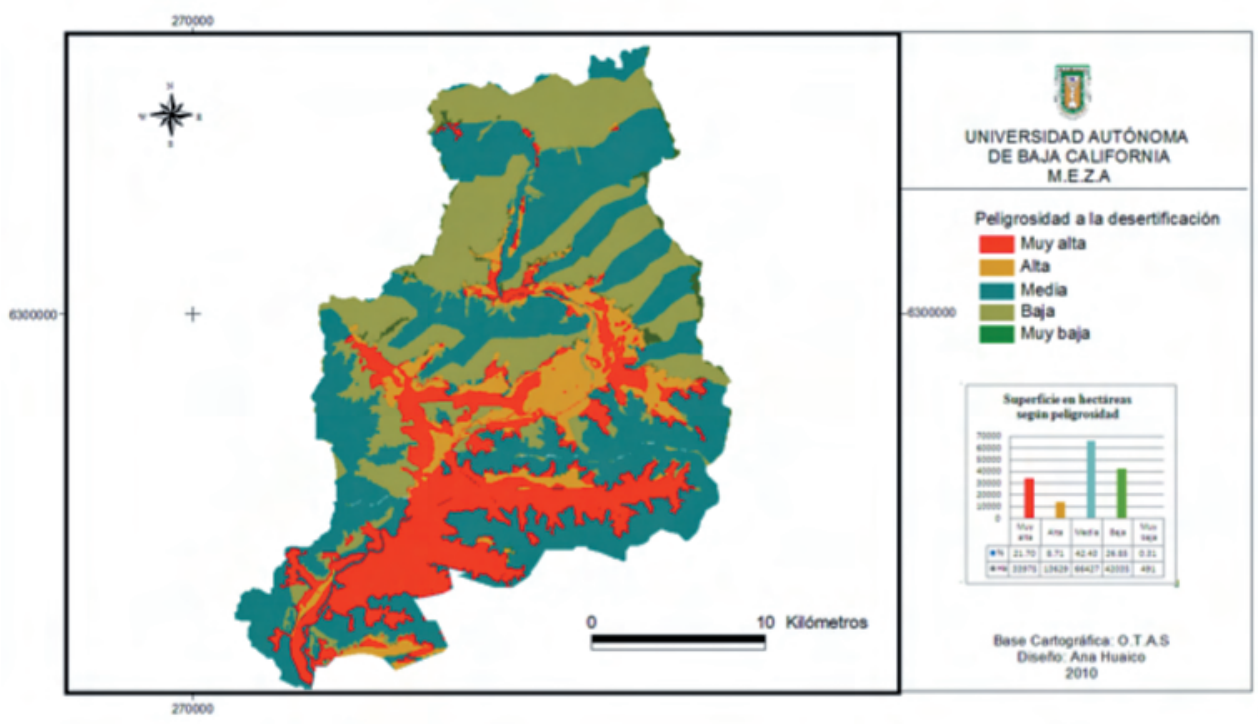

Fig. 5: El escenario actual de la cuenca del Puangue.

El escenario tendencial construido en el contexto de las presiones que actualmente posee la cuenca, entre ellos el crecimiento poblacional, la intensificación cada vez más perceptible de las actividades agrícolas, la deforestación y la alta demanda de recursos hídricos que conlleva la realización de actividades agrícolas industriales. A lo anterior se suma el contexto de cambio climático que supone una disminución de las precipitaciones y un aumento de las temperaturas, lo que podría indicar que el grado de peligrosidad aumentará con el paso de los años. La pregunta es ¿cómo se verá afectada el área de estudio, considerando el escenario actual con procesos como los que muestra la figura 4 ?
Actualmente los planes y políticas de desarrollo no contemplan el peligro de los procesos de desertificación, especialmente en el otorgamiento de los cambios de uso de suelo y la deforestación, por lo que se vislumbra que, si la situación de gestión de la cuenca continúa con su ritmo actual, esta podría tender a degradarse, lo que resultaría en un escenario con una mayor superficie en peligro de desertificación (Fig. 6).

Lo anterior indica que la cuenca tendría un $72.8 \%$ de su superficie con un alto a muy alto peligro de desertificarse por el aumento de la temperatura, disminución de las precipitaciones y con las actuales tendencias de los indicadores, como producto de la 
presión, unida a la falta de planificación de la cuenca, ya que la tendencia es continuar cambiando el uso de suelo en laderas, sin previsión de las consecuencias de los procesos erosivos por un lado, y la alta demanda de recursos hídricos, que conlleva el transformar un paisaje de vegetación principalmente de matorral, por cultivos industriales.
El diagnóstico es preocupante e indica la necesidad de gestionar el uso y manejo de los recursos naturales en la cuenca a nivel local, ya que la degradación de suelos repercute no sólo en la calidad de los recursos naturales, sino que tiene un alto impacto en la economía y desarrollo de la zona.

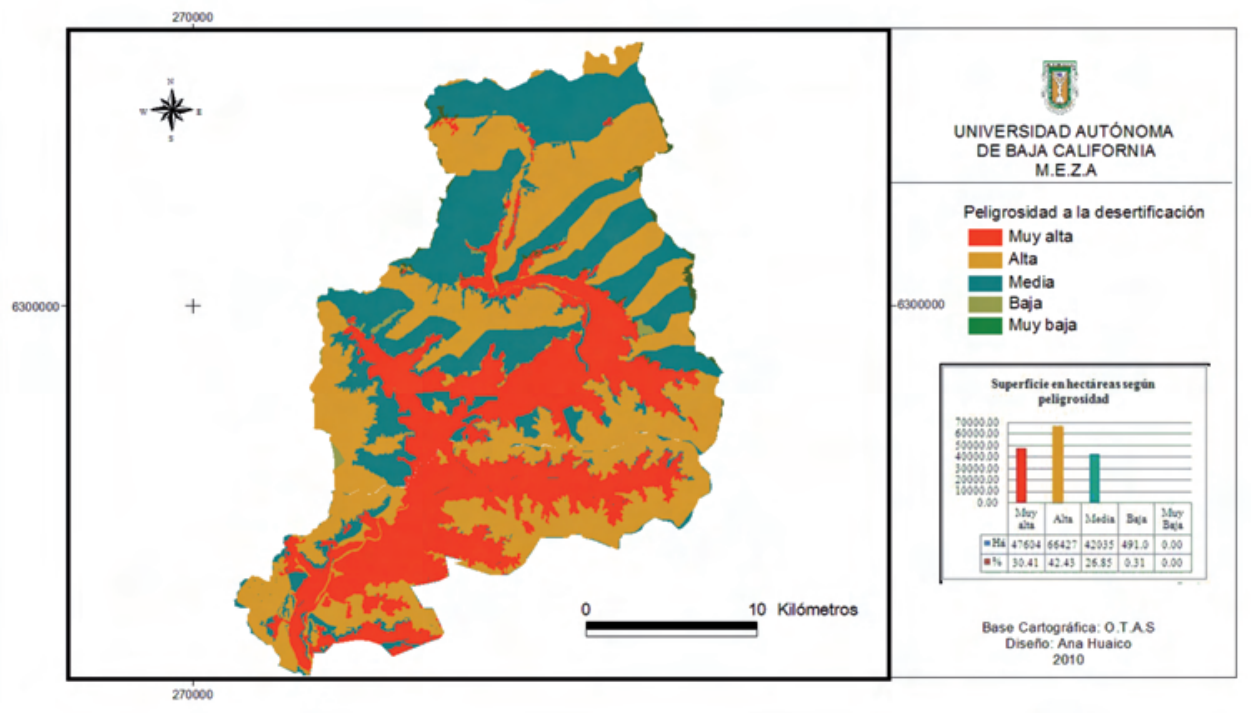

Fig. 6: Escenario tendencial de la cuenca del Puangue.

El escenario estratégico o de concertación es aquel que se aspira si se toman las medidas preventivas para que el escenario tendencial no se origine. En este caso se propone reducir la peligrosidad en sus diferentes grados, por lo que para lograr esto, se requiere que los indicadores de estado y de presión que se presentan más altos, como por ejemplo la tasa de deforestación, disminuyan a través de la gestión y manejo de los recursos.
El modelo estratégico que muestra la figura 7 busca que el $69.28 \%$ de la superficie estudiada tenga un bajo peligro a la desertificación, disminuyendo los porcentajes de peligrosidad muy alta, y manteniendo las medidas precautorias para las zonas con alta peligrosidad. 


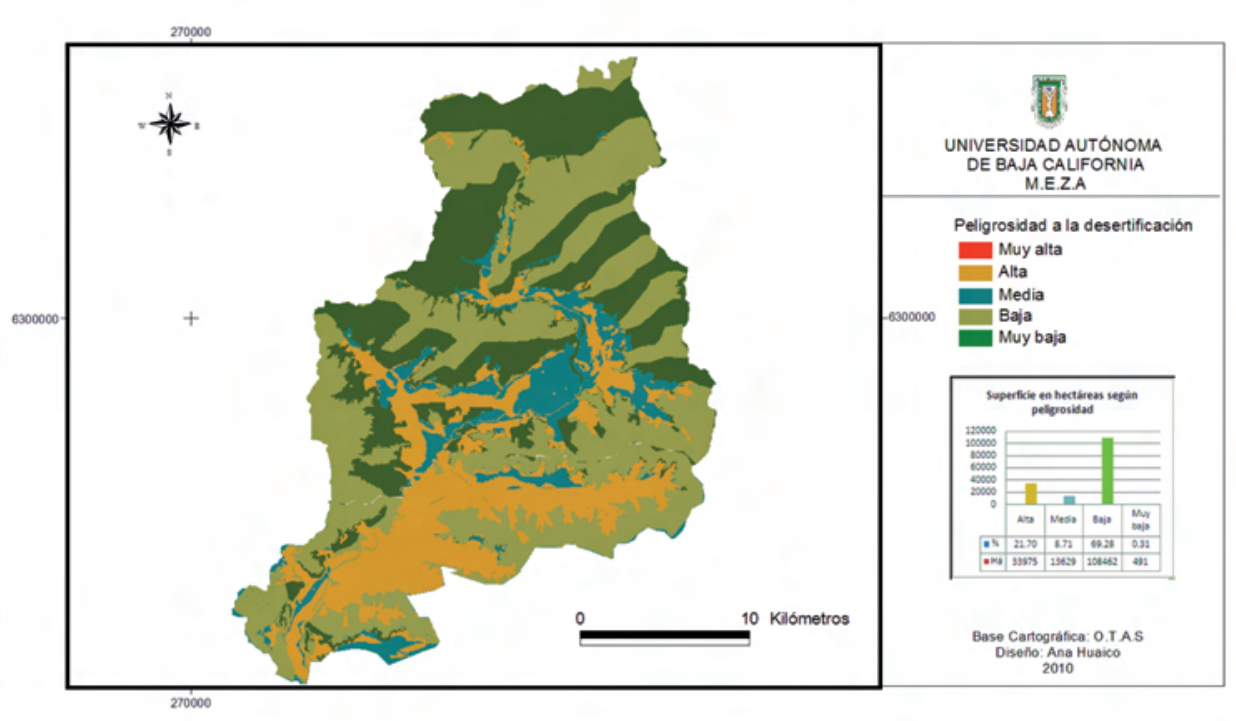

Fig. 7: Escenario estratégico o de concertación de la cuenca del Puangue.

Frente a ello se sugiere la implementación de un plan de manejo que incluya la disminución de los indicadores tales como los que tienen que ver con la gestión territorial de la cuenca, la mejora de las condiciones de pobreza especialmente en el municipio de María Pinto, mejorar las prácticas de laboreo, hacer un diagnóstico actualizado de las propiedades agrológicas de los suelos, así como también realizar un estudio acabado de la gestión del agua y contemplar la disponibilidad de agua en el otorgamiento de permisos de cambio de uso de suelo.

\section{DISCUSIÓN}

Con respecto al concepto de desertificación ha sido difícil llegar a un consenso con respecto a su definición y metodologías de diagnóstico, esto principalmente por la complejidad de los procesos que le dan origen. Es así como MAESTRE et al. (2006) menciona que por la multiplicidad de agentes que lo causan, es focalizarse en las variables críticas orientadas a entender las causas más que los efectos de la desertificación.
El presente trabajo es un ejemplo de lo anterior, ya que a partir de las propuestas de diversos proyectos y autores, $\mathrm{y}$ en función de los antecedentes provenientes de la observación de campo (como un trabajo previo al desarrollo de los indicadores), se eligieron aquellos más relevantes para el área de estudio y que reflejaban causas directas o indirectas, considerando además la disponibilidad de la información.

En este sentido es relevante mencionar que aún no se ha logrado generar una metodología sólida con respecto al tema a nivel local, nacional y más para América Latina, y hoy se cuenta con el gran desafío de unir todos los aspectos, ya sean biofísicos, socioeconómicos, de gestión y culturales que propician el fenómeno.

A nivel local los indicadores requieren adecuarse a las necesidades de la problemática observada y percibida por la propia comunidad o por el investigador, por lo que se plantea la necesidad de un trabajo de campo previo a la aplicación de los métodos de diagnóstico. 
Con respecto a la construcción de escenarios, se logró estimar en un horizonte de tiempo (50 años), cuánto aumentarán en superficies las zonas en peligro de desertificarse, por lo que la construcción de escenarios como metodología y tal como lo menciona la EEA (2001), son muy útiles, porque ofrecen un panorama de los futuros estados alternativos del medio ambiente en la ausencia de políticas ambientales adicionales (a partir de los escenarios de referencia).

\section{CONCLUSIONES}

Fue necesario realizar un trabajo previo de campo para determinar las variables críticas o indicadores y elaborar el índice de peligrosidad ambiental a la desertificación, y en función de la disponibilidad de información del área en estudio, ya que no fue posible a nivel local aplicar alguna de las metodologías preexistentes para el diagnóstico debido a la multiplicidad de causas que originan la desertificación. Las variables críticas deben de representar en lo posible directa o indirectamente las situaciones culturales y socioeconómicas del área, sin embargo se hace necesario un estudio más acabado de las relaciones del sistema humano-ambiental para la realización del plan de manejo.

Si hoy no se aplican medidas de manejo en la cuenca para prevenir los procesos de desertificación en los próximos años, se tendrán 114.031 hectáreas en peligro de desertificación y otras 42.035 hectáreas con peligrosidad media.

Debido a la falta de estudios en la materia, no fue posible llegar a determinar en cuánto tiempo llegaría a manifestar la cuenca evidencia de zonas desertificadas. Por lo mismo se hace necesario prevenir, aunque sí es posible determinar cuál es la superficie en peligro de desertificación y los escenarios futuros de la peligrosidad.
Ladefiniciónde escenariosesunaherramienta muy útil para la toma de decisiones, ya que desde un punto referencial, se definen las posibles situaciones ambientales en situaciones críticas y estratégicas, por lo que se convierte en una herramienta fundamental para la gestión y la visualización espacial de la problemática.

\section{REFERENCIAS}

ABRAHAM, E., 2003. Desertificación: bases conceptuales y metodológicas para la planificación y gestión. Aportes a la toma de decisión. Zonas Áridas, Lima. La Molina, 7:19-68.

\section{MINISTERIO DE PLANIFICACIÓN DEL GOBIERNO DE CHILE, 2006.} Encuesta de caracterización socioeconómica nacional CASEN. http://www.mideplan.cl/ casen/index.html

\section{CENTRO DE INVESTIGACIONES DE} RECURSOS NATURALES - CORFO, 1990. Atlas agroclimático de Chile. Regiones IV a la IX, publicación $N^{\circ} 87$. Santiago.

\section{CORPORACIÓN}

NACIONAL

FORESTAL, 2007. Mapa preliminar de la desertificación en Chile. http//www.conaf.cl

CONGRESO NACIONAL DE CHILE, 1994. Ley 19.300: Bases Generales del Medio Ambiente. Promulgada el $1^{\circ}$ de marzo de 1994, por el Presidente de la República Patricio Aylwin Azócar y publicada en el Diario Oficial el día 9 de marzo de 1994.

DE PEDRAZA J., 2006. Algunas consideraciones geológicas de la desertificación. Cuadernos de Sostenibilidad y patrimonio natural: Desertificación, problemática y soluciones en la España de las Autonomías, 10:21-28. 
EUROPEAN

ENVIRONMENT

AGENCY, 2001. Scenarios as Tools for International Environmental Assessments. Environmental Issue Report No 24. Office for Official Publications of the European Communities, Luxembourg. http://www.eea.eu.int.

FARAJZADEH M. \& ND NIK EGBAL, 2007. Evaluation of MEDALUS model for desertification hazard zonation using GIS; Study area: Iyzad Khast Plain, Iran. Pakistan Journal of Biological Science 10 (16): 2622-2630.

GÓMEZ OREA, D., 2001. Ordenación Territorial. Ediciones Mundi-Prensa. España.

GUERRA V. \& S. OCHOA, 2006. Evaluación espacio-temporal de la vegetación y uso del suelo en la reserva de la biosfera pantanos de Centla, Tabasco (1990-2000). Investigaciones Geográficas, 59:7-25.

GEESON N. A., C.J. BRANDT y J.B. THORNES，2002. Mediterranean Desertification: A mosaic of Processes and Responses. GEESON N. A., C.J. BRANDT y J.B. THORNES (Eds.). John Willey \& Sons.

INTERGOVERNMENTAL PANEL ON CLIMATE CHANGE, 2007. http://www. ipcc.ch/pdf/assessment-report/ar4/syr/ar4 syr_sp.pdf

INEGI, 2006. Instituto Nacional de Estadística y Geografía, México. MODELO DE PRESIÓN - ESTADO - RESPUESTA (PER) (OCDE - 2003). http://www2.inegi. gob.mx/estestint/ficha $\cdot$ asp idf $=559$

INSTITUTO NACIONAL DE ESTADÍSTICAS, 1960. Censo Población y Vivienda.
INSTITUTO NACIONAL DE ESTADÍSTICAS, 2002. Censo Población y Vivienda.

KOSMAS, C.; M. KRIKBY \& N. GEESON, 1999. Manual on key indicator of desertification and mapping environmentally sensitive areas for desertification, En: European Commission. pp. 13-65.

MAESTRE F., J. REYNOLDS, HUBERSANNWALD E. HERRICKS, J. \& M. STAFFORD, 2006. Understanding global desertification biophysical and socioeconomic dimensions of hidrology. En: D'Odorico and A. Porporato (Eds.), Dryland Ecohidrology. Millennium Ecosystem Assessment (MEA) (2005). Ecosystems Services and Human Wellbeing, Volume 2: 315-332.

MILLÁN M.; M.J. ESTRELLA; SANZ M.J.; E. MANTILLA, MARTÍN M. F. PASTOR, SALVADOR R. VALLEJO, ALONSO L., G. GANGOITI, ILARDIA J.L., M. NAVAZO, ALBIZURI A., B. ARTIÑANO,CICCIOLIP.,G.KALLOS, CARVALHO R. A., D. ANDRÉS, HOFF A., WERHAHN J., G. SEUFERT. \& B. VERSINO, 2005. Climatic Feedbacks and Desertification: The Mediterranean Model. Journal of climate American Meteorological Society, Vol.18: 684-701.

MILLENNIUM ECOSYSTEM ASSESSMENT, 2005. Ecosystems Services and Human Well-being, Volume 2, Scenarios. World Resources Institute, Washington DC. 567 p.

MINISTERIO DE VIVIENDA Y URBANISMO, 2009. Circulares División de Desarrollo Urbano, DDU. Ministerio de Vivienda y Urbanismo. http://www. minvu.cl/opensite_20070321144346. aspx\#20070321151546 
MORALES C. \& S. PARADA, 2005. Pobreza, desertificación y degradación de los recursos naturales. Libro de la Cepal, 87: 25-36.

ORDENAMIENTO TERRITORIAL AMBIENTALMENTE SUSTENTABLE, 1999. Gobierno Regional Metropolitano. Sistema de información territorial: http:// otas.gorerm.cl/gore/home.aspx

$\begin{array}{llllllll}\mathbf{S} & \mathbf{E} & \mathbf{R} & \mathbf{V} & \mathbf{I} & \mathbf{C} & \mathbf{I} & \mathbf{O}\end{array}$ AEROFOTOGRAMÉTRIOCO DE CHILE, 1955.Proyecto Aerofotogramétrico de la OEA en Chile.

SMITH K. \& D. PETLEY, 2008. Environmental hazards: assessing risk and reducing disaster. Taylor \& Francis- e Library. New York.

REYNOLDS J.F.; MAESTRE， F.T.; HUBER-SANNWALDS E.; HERRICK, J. \& P.R. KEMPS, 2005. Aspectos socioeconómicos y biofísicos de la desertificación. Ecosistemas, 14 (3): 3-21.

REYNOLDS, J.F.; STAFFORD SMITH, J.; LAMBIN, E.F.; TURNER, B.L.; MORTIMORE, II M.; BATTERBURY， S.P.J.; DOWNING, T.E.; DOWLATABADI, H.; FERNANDEZ, R.J.; HERRICK, J.E.; HUBER-SANNWALD， E.; JIANG，H.; LEEMANS, R.; LYNAM, T.; MAESTRE, F.T.; AYARZA, M. \& B. WALKER, 2007. Global desertification: Building a science for dryland development. Science, 316: 847851.

UNCED, 1992. Department of Economic and social affairs, Division for Sustainable Development. Department of Economic and social affairs, Division for Sustainable Development, UN: http://www.un.org/ esa/sustdev/documents/agenda21/spanish/ agenda 21 spchapter 12 


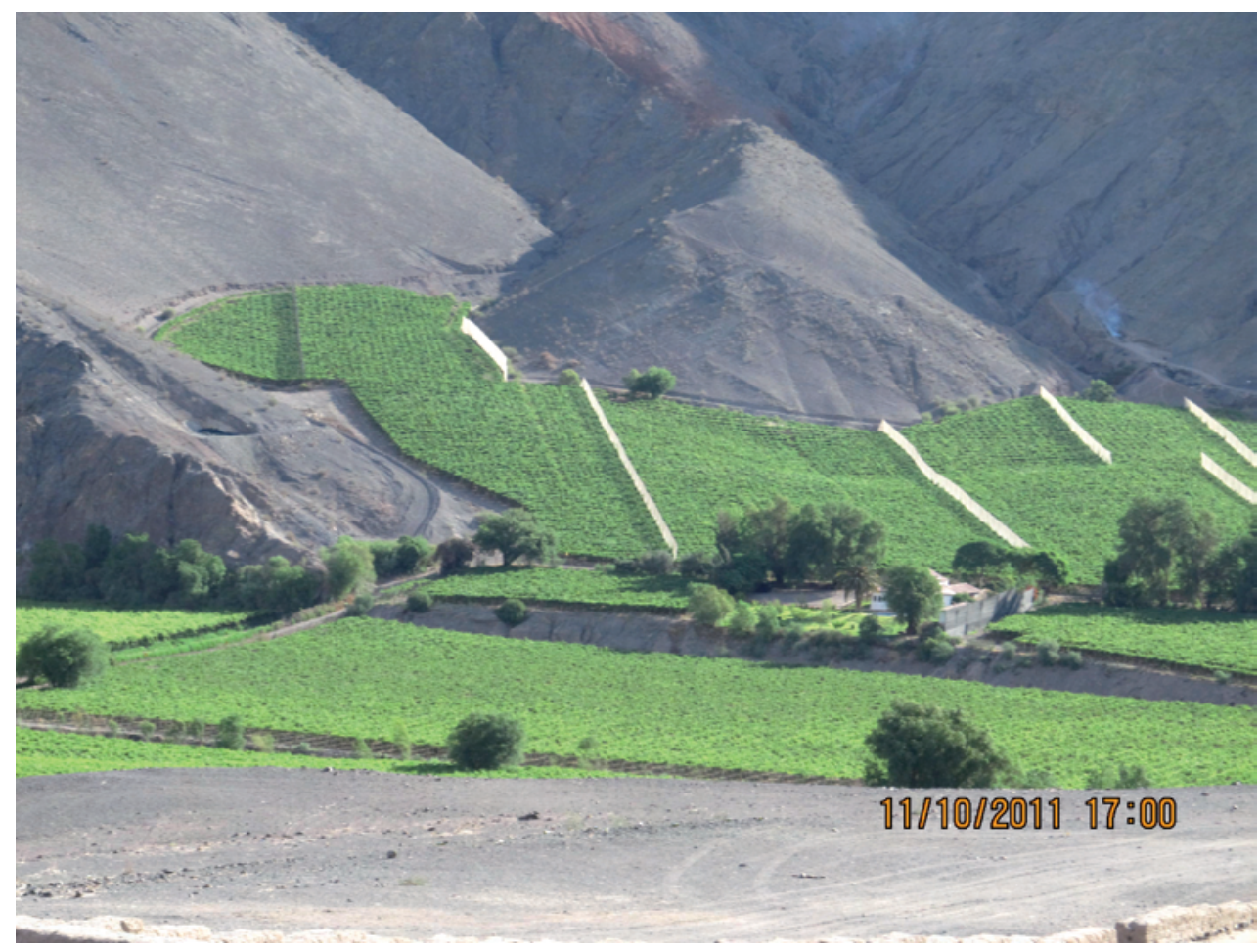

Proceso de reconversión productiva y uso intensivo del suelo con parronales, en ambiente de desierto marginal. Sección media del valle del río Copiapó, Región de Atacama. Excursión COMLAND UGI-DEPARTAMENTO DE GEOGRAFÍA, UNIVERSIDAD DE CHILE. Noviembre 2011. Fig. y texto, Joselyn Arriagada González (joarriag@uchilefau.cl). 